\title{
Kleines Lexikon der Rheumatologie
}

Zusammenfassend die werden hier in Kürze nochmals die wichtigsten rheumatischen Erkrankungen erläutert.

Fibromyalgie: Fibros (griech.) = Faser, Myos (griech.) = Muskel und Algos (griech.) = Schmerz. Begriff für einerheumatischeErkrankung, diesehr starkeSchmerzen im Bereich der Muskel- und Sehnenansatzpunkte verursacht. Sie ist häufig schwer zu behandeln.

Gicht: Rheumatische Erkrankung, die durch zu viel Harnsäure im Blut und im Gelenk verursacht wird. Vor allem Patienten, die zu viel Fleisch essen, können darunter leiden, denn Fleisch ist ein Hauptlieferant von Verbindungen, deren Stoffwechselendprodukt Harnsäure ist. Gicht verursacht stärkste und plötzliche Schmerzzustände, ist jedoch meist gut zu behandeln.

Juvenile Arthritis: Juvenus (lat.) = jugendlich, Arthros (griech.) = Gelenk und -itis = Entzündung. Gelenkentzündung im jugendlichem Alter. Ein Sammelbegriff für entzündliche Gelenkerkrankungen im Kindes- und Jugendalter.

Lyme-Borreliose: Borrelien = Bakterienart, - ose $=$ Krankheit, also eine Borrelienerkrankung. Sie wird durch Zecken (Holzbock) übertragen. Lyme ist ein Ort in den USA, wo diese Infektion gehäuft auftrat. Die Borreliose ist schwierig zu erkennen, aber gut zu behandeln.

Mischkollagenosen/undifferenzierte Kollagenosen: Bindegewebserkrankung, die als Überlappungssyndrom mit Symptomen, die im Einzelnen verschiedenen entzündlich-rheumatischen Erkrankungen entsprechen können, auftritt. Verschiedene Organsysteme wie z.B. Herz, Gelenke, Nieren und Lungen können betroffen sein.

Morbus Bechterew (Synonym ankylosierende Spondylitis): Morbus (lat.) = Krankheit, Bechterew = russischer Arzt, der diese Erkrankung erstmals ausführlich als eigenständige Erkrankung beschrieben hat. Morbus Bechterew ist eine entzündliche Wirbelsäulenverkrümmung, die schon im frühen Erwachsenenalter auftritt. Erste Warnhinweise sind tiefsitzende Rückenschmerzen, von denen der Patient frühmorgens wach wird. Rechtzeitig erkannt, insbesondere durch Krankengymnastik und Physiotherapie gut behandelbar. 
Morbus Reiter: Reiter = deutscher Militärarzt, der diese Erkrankung erstmals bei einem jungen Soldaten beschrieben hat. Morbus Reiter ist eine entzündliche Erkrankung unter Beteiligung der Gelenke, der Haut und der Augen. Verursacht wird sie durch Bakterien, die entweder über den Magen-Darm-Trakt oder das urogenitale System in den Körper eindringen. Deshalb ist ein weiteres Symptom entweder Durchfall oder Ausfluss aus der Harnröhre. Gut behandelbar.

Myositis: Myos (griech.) = Muskel, -itis = Entzündung, also Muskelentzündung. Es gibt in der Rheumatologie verschiedene Formen von Muskelentzündungen (Dermato- und Polymyositis). Der Patient fühlt sich abgeschlagen, hat starke Muskelschmerzen und Lähmungen in bestimmten Körperregionen, etwa wie bei einem sehr schweren Muskelkater. Myositis ist schwierig zu behandeln und muss unbedingt vom Spezialisten betreut werden.

Osteoporose: Die Osteoporose ist eine systemische Skeletterkrankung, die mit einer erniedrigten Knochendichte und Veränderung der Mikroarchitektur einhergeht. Daraus resultiert ein erhöhtes Frakturrisiko.

Polymyalgia rheumatica/Arteriitis temporalis: Poly- (griech.) = viel, zahlreich, my- von myos (griech.) = Muskel, - algia von algos (griech.) = Schmerz. Rheumatische Erkrankung, die vorwiegend ältere Menschen betrifft. Frauen erkranken dreimal so häufig wie Männer. Die Polymyal gia rheumatica geht mit allgemeinen Symptomen und Muskelschmerzen einher. Verursacht wird sie durch eine Gefäßentzündung.

Psoriasis: Psora (griech.) = Krätze, gemeint ist dieSchuppenflechte. Diese kann gelegentlich zusammen mit entzündlichen Gelenkbeschwerden auftreten und muss dann unbedingt von einem Rheumatologen behandelt werden, weil die Gelenke sonst zerstört werden könnten.

Raynaud-Syndrom: Raynaud = französischer Arzt, Erstbeschreiber einer Durchblutungsstörung im Bereich der Finger. Vor allem bei Kälte werden die Finger zunächst weiß, dann blau und schließlich rot. Dieses Symptom tritt bei vielen entzündlich-rheumatischen Erkrankungen auf.

ReaktiveArthritis: Gelenkentzündung, die durch die (Fehl-)Reaktion des I mmunsystems auf eine bakterielle Infektion verursacht wird. Richtig erkannt, ist sie gut zu therapieren.

Rheumatoide Arthritis (RA): Chronische, gelenkschädigende, entzündlich-rheumatische Erkrankung. Im Volksmund als «Gelenk-Rheuma» bekannt. 
Rheumatisches Fieber: Nach einer bakteriellen Infektion kommt es zu einer überschießenden Abwehrreaktion des Körpers, die dazu führen kann, dass die Nieren, die Gelenke und das Herz angegriffen werden. In der Folge kann es zur Zerstörung der Herzklappen kommen. Diese Erkrankung tritt fast immer in der Kindheit auf. Da heutzutage Antibiotika überall verfügbar sind, ist rheumatisches Fieber in Deutschland sehr selten geworden.

Sjögren-Syndrom: Henrik Sjögren, schwedischer Augenarzt, fasste die klinische Erkrankung bzw. die Syndrome der trockenen Bindehautentzündung und der Mundtrockenheit erstmals 1933 als ein eigenständige Erkrankung zusammen. Das Sjögren-Syndrom ist eine chronisch-entzündliche Bindegewebserkrankung, die zur Einschränkung der Drüsenproduktion führt. Vorwiegend betroffen sind Tränen- und Speicheldrüsen. Zusätzlich können begleitend anderer Organsysteme betroffen werden.

Sklerodermie: Skleros (griech.) = hart, derb, Derma ( griech.) = Haut, also eineVerhärtung der Haut. Es gibt verschiedene Arten der Sklerodermie. Durch die Entzündung des Bindegewebes kommt es zu einer narbigen Umwandlung des betroffenen Gewebes. Wenn z.B. die Lunge betroffen ist, kommt es auf Dauer zum Funktionsverlust, und es entsteht Atemnot. Sklerodermie ist eine schwere rheumatische Erkrankung und muss unbedingt vom Spezialisten betreut werden.

Systemischer Lupus erythematodes (SLE): Der SLE ist eine entzündlich-rheumatische Erkrankung, die häufiger Frauen als Männer befällt. Es können verschiedenen Organsysteme (Nieren, Lunge, Gelenke) betroffen sein. Typisch ist das so genannte Schmetterlingser ythem. Diagnostisch sind, neben den klinischen Symptomen, Autoantikörper wegweisend.

Vaskulitis: Vasa (lat.) = Gefäß, -itis = Entzündung. DieVaskulitis ist eine Gefäßentzündung. Vaskulitis ist ein Sammel begriff für vieleverschiedenen Entzündungsformen. Zum Beispiel werden Gefäßentzündungen der kleinen, mittelgroßen und großen Gefäßeunterschieden. Die Symptomeentsprechen den Funktionsstörungen der Organe, die durch entzündete Gefäße betroffen sind. 